\title{
Unidentified emission features in the R Coronae Borealis star V854 Centauri
}

\author{
L. C. Oostrum ${ }^{1,2}$, B. B. Ochsendorf ${ }^{3}$, L. Kaper ${ }^{1}$, and A. G. G. M. Tielens ${ }^{4}$ \\ 1 Anton Pannekoek Institute for Astronomy, University of Amsterdam, PO Box 94249, 1090 GE Amsterdam, The Netherlands \\ e-mail: 1.c.oostrum@uva.nl \\ 2 ASTRON, The Netherlands Institute for Radio Astronomy, PO Box 2, 7990 AA Dwingeloo, The Netherlands \\ ${ }^{3}$ Department of Physics and Astronomy, The Johns Hopkins University, 3400 North Charles Street, Baltimore, MD 21218, USA \\ ${ }^{4}$ Leiden Observatory, Leiden University, PO Box 9513, 2300 RA Leiden, The Netherlands
}

Received 11 July 2017 / Accepted 2 December 2017

\begin{abstract}
During its 2012 decline, the R Coronae Borealis star (RCB) V854 Cen was spectroscopically monitored with X-shooter on the ESO Very Large Telescope. The obscured optical and near-infrared spectrum exhibits many narrow and several broad emission features, as previously observed. The envelope is spatially resolved along the slit and allows for a detailed study of the circumstellar material. In this Letter, we report on the properties of a number of unidentified visual emission features (UFs), including the detection of a new feature at $8692 \AA$. These UFs have been observed in the Red Rectangle (RR), but their chemical and physical nature is still a mystery. The previously known UFs behave similarly in the RR and in V854 Cen, but are not detected in six other observed RCBs. Some hydrogen might be required for the formation of their carrier(s). The $\lambda 8692$ UF is present in all RCBs. Its carrier is likely of a carbonaceous molecular nature, presumably different from that of the other UFs.
\end{abstract}

Key words. circumstellar matter - stars: individual: V854 Cen

\section{Introduction}

$\mathrm{R}$ Coronae Borealis stars (RCBs) are rare, hydrogen-deficient supergiants that exhibit strong declines in their brightness (Clayton 2012). Only about 100 RCBs are known in the Galaxy. Their rarity is an indication of a short evolutionary phase and/or points to a peculiar mode of stellar evolution: (i) a merger between a $\mathrm{CO}$ and $\mathrm{He}$ white dwarf, or (ii) a final helium-shell flash leading to the expansion to supergiant size (Iben et al. 1996; Saio \& Jeffery 2002). The decline is thought to be due to the formation of clouds of carbon dust along the line of sight, obscuring the stellar photosphere so that the circumstellar envelope becomes observable in emission. Such a natural coronograph provides a unique opportunity for studying the chemical and physical nature of the circumstellar envelope of these peculiar objects.

Owing to the irregularity of these events, limited spectra are available of RCBs in decline. V854 Cen is an RCB that is of particular interest because it is one of the few RCBs that include hydrogen lines in their spectra; it is the most hydrogen-rich RCB after DY Cen (Asplund et al. 1998; Jeffery \& Heber 1993). V854 Cen and DY Cen are also the only RCBs in which polycyclic aromatic hydrocarbons (PAHs) have been detected (García-Hernández et al. 2011a). The spectra include the weak $18.9 \mu \mathrm{m}$ band, which is now generally attributed to $\mathrm{C}_{60}$ (Cami et al. 2010; Sellgren et al. 2010). Moreover, V854 Cen shows some unidentified visual emission features (UFs) in its decline spectrum (Rao \& Lambert 1993b). These UFs have only been detected in the Red Rectangle proto-planetary nebula (RR; Schmidt \& Witt 1991). In that object, the UFs change in shape, intensity, and peak position as a function of position in the nebula (Van Winckel et al. 2002; Wehres et al. 2011). Additional impetus for a study of the visual emission features is provided by the potential link between the visual emission bands in the $\mathrm{RR}$ and the diffuse interstellar band (DIB) absorption features in the interstellar medium (Sarre et al. 1995).

In this Letter, we show for the first time the spatial structure of the UFs in V854 Cen during its 2012 decline. We compare the characteristics of these features to those detected in the RR. In addition, we search for new emission features (300-2500 nm) in V854 Cen, as well as in six other RCBs.

\section{Observations and data reduction}

Time on the ESO VLT was granted for observing V854 Cen within a window of 4 months in 2012. The object was monitored by the American Association of Variable Star Observers (AAVSO). When the star's visual magnitude dropped below $m_{\mathrm{v}}=8$ (maximum-light $m_{\mathrm{v}}=7.1$ ), multiple spectra were obtained with VLT/X-shooter (Vernet et al. 2011) during the decline that lasted around 2.5 months. Additionally, in 2013, $\mathrm{X}$-shooter spectra were taken of V854 Cen and a few other RCBs, known to be in decline as determined from their AAVSO light curves, with the aim to search for UFs. V854 Cen was then at maximum light. For all observations, the highest resolution mode was used, where $R \approx 10000,18000$, and 11500 for the UVB, VIS, and near-infrared (NIR) arms, respectively. An observation log is given in Table 1 .

The spectra were reduced using the $\mathrm{X}$-shooter pipeline version 2.2.0 (Modigliani et al. 2010) and flux calibrated using spectrophotometric standard stars. Telluric correction of the 1D NIR spectra was made with Spextool (Vacca et al. 2003) using telluric standard spectra obtained at similar airmass and close in time to the targets. The resulting spectra were shifted to the rest-frame of the observed source. 


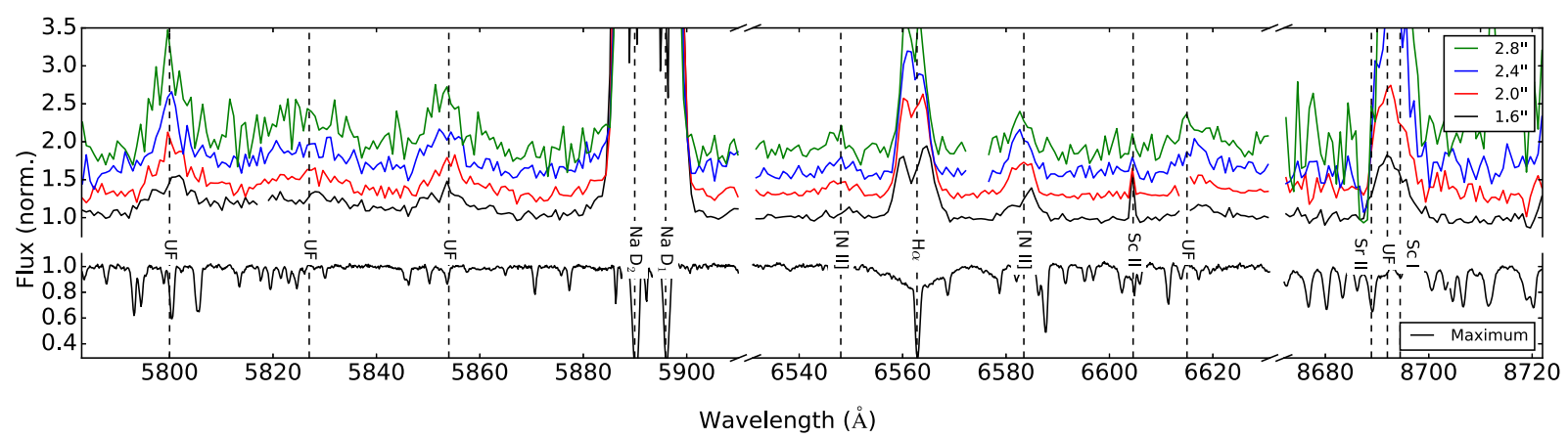

Fig. 1. V854 Cen decline spectra at different offsets from the central object (see inset) and at maximum light (bottom spectrum). During a decline, the absorption spectrum converts into an emission spectrum, the strongest emission lines being the Na I D doublet. The progressive eclipsing of narrow emission lines during the decline, but not the broad lines, implies that the broad line emission is more extended (Clayton 1996). Additionally, a sequence of unidentified emission features is clearly detected between 5800 and $5860 \AA$, at $6617 \AA$, and at $8692 \AA$. The narrow Y II $\lambda 6614$ emission line has been removed from the $1.6^{\prime \prime}$ and $2.0^{\prime \prime}$ offset spectra for plotting purposes. The absorption lines near 5800 and $5854 \AA$ are due to C I and Ba II, respectively.

Table 1. Log of VLT/X-shooter observations.

\begin{tabular}{lllllll}
\hline \hline Target & $\begin{array}{l}d \\
(\mathrm{kpc})\end{array}$ & Phase & Obs. date & $\begin{array}{l}m_{\mathrm{v}} \\
(\mathrm{max})\end{array}$ & $\begin{array}{l}m_{\mathrm{v}} \\
(\mathrm{obs})\end{array}$ & $S / N$ \\
\hline V854 Cen & 2.4 & Max & $2012-05-04$ & $7.1^{1}$ & 7.2 & 90 \\
& & Min & $2012-06-14$ & & 13.3 & 70 \\
& & Min & $2012-06-18$ & & 12.8 & 130 \\
& & Rise & $2012-07-05$ & & 9.9 & 170 \\
& & Rise & $2012-07-05$ & & 9.9 & 150 \\
& & Rise & $2012-07-05$ & & 9.9 & 170 \\
ASAS-RCB-12 & 17.5 & Min & $2013-07-15$ & & 7.2 & 85 \\
R CrB & 1.3 & Min & $2013-07-15$ & $5.7^{3}$ & 13.7 & 35 \\
RT Nor & 9.9 & Min & $2013-07-15$ & $10.6^{3}$ & 15.2 & 20 \\
RZ Nor & 10.5 & Min & $2013-07-15$ & $10.6^{3}$ & 16.2 & 15 \\
S Aps & 7.1 & Min & $2013-07-15$ & $9.6^{3}$ & 14.3 & 10 \\
V CrA & 6.6 & Min & $2013-07-15$ & $9.4^{3}$ & 17.5 & 10 \\
\hline
\end{tabular}

Notes. During the rise of V854 Cen, three different position angles were used. The magnitudes were measured from the acquisition images, except for V854 Cen during maximum light, for which they are saturated. For these, values from the AAVSO were used. The distance of V854 Cen is based on the $(V-I)-M_{V}$ relation for RCBs in the LMC by Tisserand et al. (2009). For the others, a typical value of $M_{\mathrm{v}}=-5$ (Clayton 2012) is assumed. The signal-to-noise ratio was measured in the continuum around $6200 \AA$.

References. (1) Samus' et al. (2003); (2) Tisserand et al. (2013); (3) Ducati (2002).

\section{Unidentified emission features}

\subsection{Detected bands}

A section of the X-shooter spectrum of V854 Cen is shown in Fig. 1. The broad features at 5800, 5827, 5854 and $6617 \AA$ were first recognized in V854 Cen by Rao \& Lambert (1993b) during a deep decline $\left(m_{V} \sim 15\right)$. The similarity between these features and the emission features in the RR was noted before. We expand upon this by considering the spatial and dynamical (i.e. radial velocity) structure of the UFs in V854 Cen. None of these bands is detected in any of the other observed RCBs. The spectra reveal a broad emission feature at $8692 \AA$ that has not been seen before in any RCB, nor in the RR.

Of the seven bands, only the $\lambda \lambda 5800,5827,5854$, and 6617 bands are detected in the 2012 decline of V854 Cen. The absence of three of the features (at 5772, 6774, and $6997 \AA$ ) may be attributed to the depth of the decline, as this decline is two magnitudes - or a factor $\sim 6$ in flux - shallower than the 1992 decline, and the non-detected features are the weakest ones. The four detected bands are present in the two spectra taken during the deepest part of the decline $\left(m_{V} \sim 13\right)$, just beyond the stellar continuum along the slit. The features are strongest in the spectrum taken just after the minimum, on 2012-06-14. Only in that spectrum is the signal-to-noise ratio $(\mathrm{S} / \mathrm{N})$ in the features high enough to allow for an analysis of their spatial distribution. The features are detected at different distances from the central object, as shown in Fig. 1, which also shows the maximum light spectrum for comparison. They are only very marginally detected at the western side of the star (negative offsets), hence we focus on the eastern side (positive offsets). None of the bands are detected in the other RCBs.

The whole wavelength range of X-shooter (300-2500 nm) has been searched for emission features. One new feature was detected, which is present in all V854 Cen spectra, at $\sim 8692 \AA$. It is also detected in five of the other six observed RCBs in decline (Fig. 2). Similar to many features associated with the circumstellar material, this feature is only detected off-source. The feature is blended with two photospheric lines: Sr II $\lambda 8689$ and Sc I 18694. In order to separate the nebular features from the stellar ones, the off-source spectrum was divided by the onsource spectrum and the resulting spectrum was renormalised. The presence of the feature during maximum-light is especially noteworthy.

\subsection{Spatial and dynamical structure of the bands}

As the circumstellar envelope of V854 is spatially resolved along the spectrograph slit, position-velocity (PV) diagrams can be produced to study the kinematic structure of the emission lines as a function of the distance from the star. In Fig. 3 we show the dynamical structure as revealed by the $\mathrm{Ca}$ II $\mathrm{K}$ resonance line, and UF8692. The other UFs are too weak to construct a PV diagram. Ca II K shows a structure suggesting that the emission is produced in roughly a shell with a radius of about $3.5^{\prime \prime}$ that expands with a velocity of $250 \mathrm{~km} \mathrm{~s}^{-1}$. The size of the shell is based on the farthest position where the flux is more than $3 \sigma$ above the noise. PV diagrams of more extended features, confirming the size and velocity of the shell, are shown in Appendix A. The shape of the UF8692 extended emission is different from the shell traced by Ca II K. It shows a change in wavelength and width for different distances from the central 


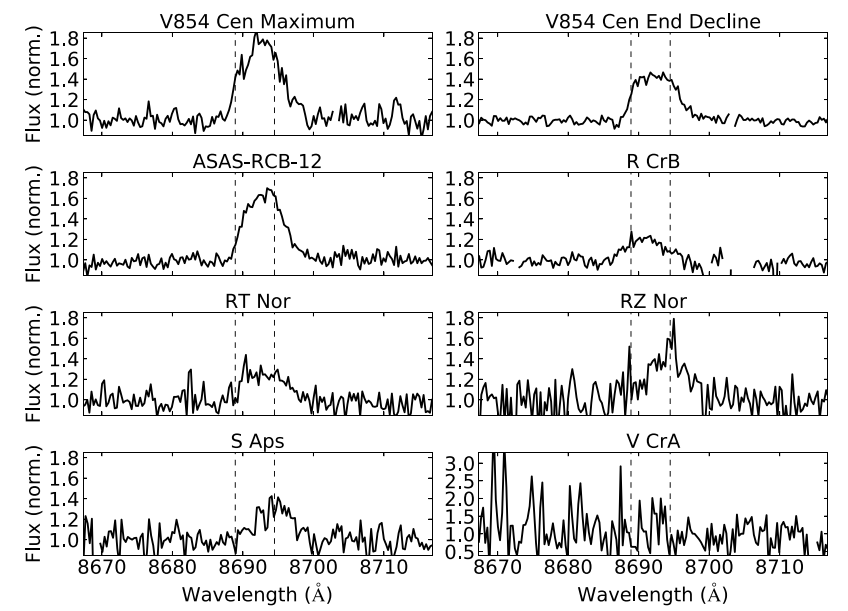

Fig. 2. $\lambda 8692$ unidentified feature (UF) in all observed RCBs. The two dashed lines indicate the position of the Sr II $\lambda 8689$ and Sc I $\lambda 8694$ photospheric lines. The UF is detected in all except $\mathrm{V}$ CrA. The position and intensity of the feature clearly vary between objects. In V854 Cen, the feature is also detected during maximum light.

object. For V854 Cen, it shifts toward the red at the eastern side of the star (i.e. positive offsets), and to the blue at the western side of the star. Additionally, it narrows with increasing distance to the star on the eastern side. It is also worth noting that the equivalent width $\left(W_{\mathrm{eq}}\right)$ of the feature - here defined such that an emission feature has a positive $W_{\text {eq }}$ - increases with distance to the star. This confirms that this newly found UF originates in the circumstellar material.

The behaviour of the $\lambda 8692$ feature is different for each RCB. In RT Nor and RZ Nor, the width of the feature does not change significantly, while a narrowing with increasing distance is observed in the other RCBs. The position of the feature shifts in all objects. There is no clear pattern to this; in some objects, only blueshift is observed, and in others, redshift and/or no shift on one side of the star. As the RCBs have different distances, different physical scales were probed, but we did not find a correlation between the behaviour of this feature and the respective distances to the RCBs.

The shape of the bands, when integrated over an interval along the slit, is well described by either one or two Gaussians. All features in the $\lambda 5825$ complex were measured together, as those bands are too close in wavelength to be considered separately. For the band at $5800 \AA$, two Gaussians were used to account for the asymmetric band shape, which significantly improved the fits. For the other bands, a single component was used. All narrow emission lines, originating from regions close to the star $\left(\sim 2 R_{*}\right.$; Clayton 1996) instead of the large-scale circumstellar material, were removed from the spectra before the fitting procedure.

For all five detected bands, the measured peak position as function of distance to the star is shown in Fig. 4 (black squares). All features (at 1.6" and 2.8") are shown in Appendix B. The narrow component in the $5800 \AA$ feature (Fig. 1) is used for the band position, as this component is stronger and dominates the peak position. The same bands in the RR are known to show a blueshift with increasing distance to the central binary system (Van Winckel et al. 2002; Wehres et al. 2011). The same analysis method as for V854 Cen was applied to the RR spectra, except for the number of components: the higher S/N RR spectra required two components for the $\lambda 5854$ and $\lambda 6617$ bands in addition to $\lambda 5800$, whereas one component was sufficient for the bands in V854 Cen. The only RR spectrum covering the
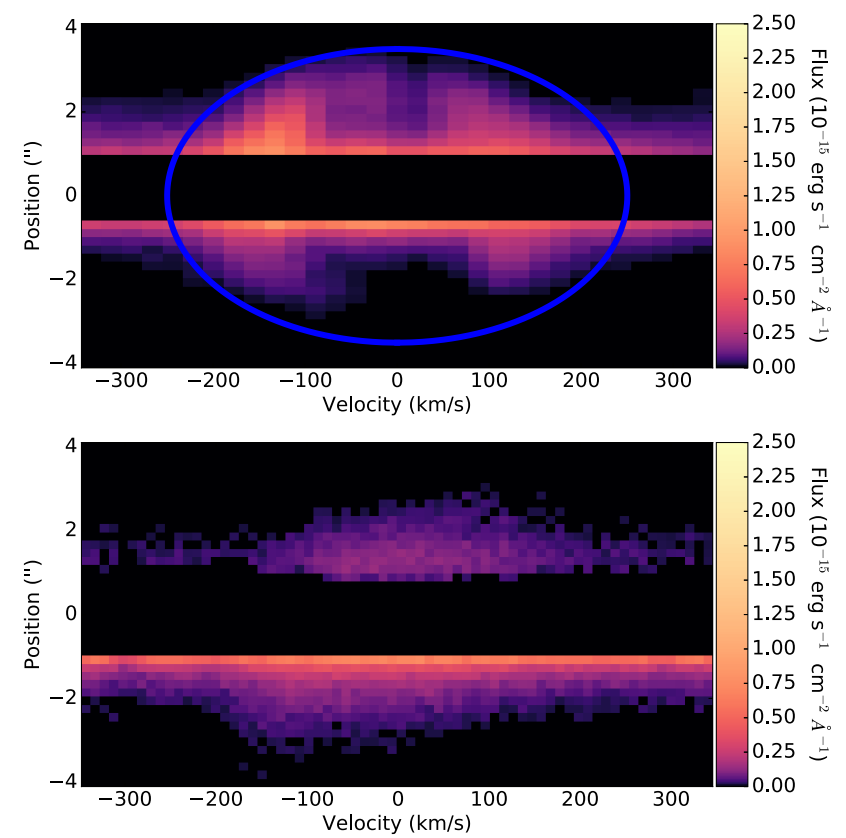

Fig. 3. Position along the spectrograph slit (vertical direction) against radial velocity for the Ca II K resonance line (top) and UF at $8692 \AA$ (bottom) in V854 Cen. The continuum flux has been subtracted, and all flux within $0.7^{\prime \prime}$ of the central object has been set to zero to enhance the visibility of these extended features. The thick blue line shows the expected maximum radial velocity at each position for a spherical shell of radius $3.5^{\prime \prime}$ and expanding with a velocity of $250 \mathrm{~km} \mathrm{~s}^{-1}$. The unidentified emission feature at $8692 \AA$ is spatially extended as well.

$\lambda 8692$ feature is from the ESPaDOnS spectrograph mounted on the CFHT, and unfortunately, it has insufficient $\mathrm{S} / \mathrm{N}$ in that wavelength region (N. L. J. Cox, priv. comm.). The measured RR band positions agree well with those provided by Wehres et al. (2011). With the exception of $\lambda 8692$, all V854 Cen and RR bands show a shift towards shorter wavelengths with increasing distance to the central object.

The RR bands show a correlation between their position and width: some bands become narrower as they shift to shorter wavelengths (Schmidt \& Witt 1991; Sarre et al. 1995; Van Winckel et al. 2002; Wehres et al. 2011). We are unable to confirm such a correlation in the V854 Cen features. It should be noted that in the RR, the features change most rapidly close to the central binary. The distance to V854 Cen is roughly three times that of the RR (710 pc; Men'shchikov et al. 2002). We therefore cannot exclude a width-wavelength correlation in the inner regions of the circumstellar material.

\section{Discussion}

We detected several emission features in the nebula surrounding V854 Cen, up to $3.5^{\prime \prime}$ or $\sim 8500$ AU from the central object. This is the farthest detection of the nebula so far. The broad emission lines from Ca II K, H $\alpha$, and [C I] 29850 show a roughly spherical outflow with velocities of up to $250 \mathrm{~km} \mathrm{~s}^{-1}$. Extended emission has been detected before up to $2.5^{\prime \prime}$ from the star in C II, with a profile suggesting higher density in the outer part of this circumstellar material (Clayton \& Ayres 2001). In addition to these atomic lines, we detected six UFs, including a new feature at $8692 \AA$. The width of the emission features is comparable to that of other broad emission lines. The UFs that were also detected in the RR have a similar width, even though the velocities in the RR are much lower $\left(\sim 7 \mathrm{~km} \mathrm{~s}^{-1}\right.$ in CO; Jura et al. 1995) than in V854 Cen. This indicates that the features are intrinsically broad. 

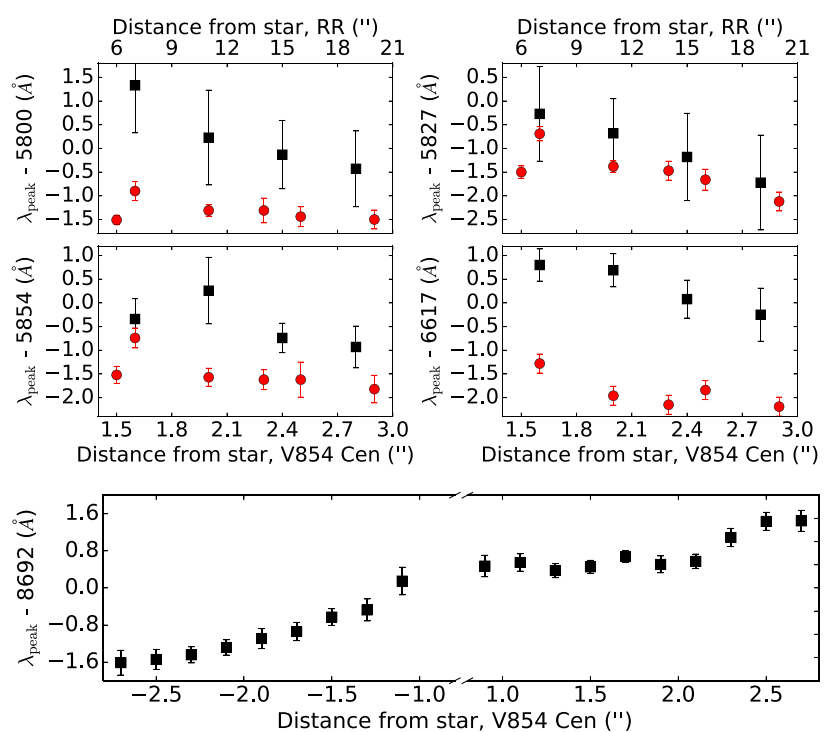

Fig. 4. Band positions of the unidentified emission features as function of distance to the central object for V854 Cen (black squares) and the Red Rectangle (red circles). There is no measurement of the $\lambda 6617$ feature at $6^{\prime \prime}$. Error bars reflect $1 \sigma$ errors. All RR bands are blueshifted with respect to the corresponding bands in V854 Cen. The 18692 band is only covered in V854 Cen and is detected on both sides of the star.

They are not Doppler broadened, unlike the broad atomic emission lines, as in that case they would be much wider than the RR features because of the high velocities in V854 Cen.

The observed shift of $\sim 1 \AA$ in the features at 5800-5854 $\AA$ corresponds to $\sim 50 \mathrm{~km} \mathrm{~s}^{-1}$. This shift may be due to rotational cooling, as in the RR (Wehres et al. 2011), however, when we assume no cooling, it is an upper limit on the radial velocity of the carrier. This velocity is significantly lower than that of the gas traced by the broad emission lines, hence the carriers are not outflowing with the high-velocity gas. There is evidence for low-velocity material in RCBs, however, when we assume that the minimum-light optical spectrum is dominated by scattered light from the central object (García-Hernández et al. 2011b). This low-velocity material is the most likely environment for the carrier of the UFs. There is also evidence for high-velocity dust around RCBs (Clayton et al. 2013), but apparently, the carriers of these features are not mixed in with this high-velocity material, possibly because the timescale or physical conditions were not conducive to their formation. The absence of the features in other RCBs may indicate that some hydrogen is required for their formation.

The $\lambda 8692$ feature may provide insight into the geometry of this material in V854 Cen, as it is the only UF that is detected clearly on both sides of the star. If we interpret the shift in this feature as a Doppler shift, it is consistent with a bipolar outflow that is being accelerated to $\sim 50 \mathrm{~km} \mathrm{~s}^{-1}$. A bipolar geometry has been suggested in the literature (e.g. Rao \& Lambert 1993a; Chesneau et al. 2014). If the other UFs are part of the same outflow, we would expect them to show a shift of $\sim 0.3 \AA$ between their closest and farthest detection. We are unable to confirm this, however, as this putative shift is smaller than the detected shifts in both V854 Cen and the RR. By the same argument as for the other features, the carrier of $\lambda 8692$ is presumably not located in the high-velocity outflow observed in the broad atomic lines.

For the observed bands, the shifts in the RR are consistent with a change in excitation temperature of a molecule (Wehres et al. 2011). For V854 Cen, we would also expect such a shift as the temperature in the outflow decreases.
Care has to be taken when comparing UF8692 to the other features, as it is the only feature present in all spectra and the only one that is detected in other RCBs. It is thus unlikely that this feature has the same carrier as one of the other emission bands. The presence of the feature in more hydrogen-deficient RCBs does indicate that the carrier is hydrogen poor. A molecular nature seems likely as the shape of the extended emission is different from that of the atomic lines, and we have not been able to find an atomic line that could reasonably be the carrier of the line. Given the high carbon abundance of RCBs and the detection of multiple carbonaceous molecules in these stars, the molecule is likely of a carbonaceous nature. The different behaviour of the feature in different RCBs is quite puzzling. It may be an indication of varying physical and chemical conditions in the complex circumstellar envelopes of RCBs.

It has been suggested that the UFs in the RR are the emission equivalent of the DIBs (e.g. Scarrott et al. 1992). The inverted DIB spectrum does show correlations with some UFs. We are unable to identify a possible DIB counterpart for the $\lambda 8692$ feature. The closest known DIB is located at 8648.3 $\AA$ (online catalogue $^{1}$ and N. L. J. Cox, priv. comm.), which is too far off to be the likely absorption equivalent of the $\lambda 8692$ feature.

Acknowledgements. The authors thank Nadine Wehres and Hans van Winckel for providing us the Red Rectangle spectra. We thank our referee Geoff Clayton for suggestions that helped improve the Letter. We acknowledge the variable star observations from the AAVSO International Database contributed by observers worldwide, and used in this research. L.C.O. would like to thank Nick Cox for discussion about DIBs and the Red Rectangle. L.C.O. acknowledges funding from the European Research Council under the European Union's Seventh Framework Programme (FP/2007-2013)/ERC Grant Agreement No. 617199. Based on observations collected under ESO programmes 089.D-0937(A) and 091.C-0934(B).

\section{References}

Asplund, M., Gustafsson, B., Rao, N. K., \& Lambert, D. L. 1998, A\&A, 332, 651 Cami, J., Bernard-Salas, J., Peeters, E., \& Malek, S. E. 2010, Science, 329, 1180 Chesneau, O., Millour, F., De Marco, O., et al. 2014, A\&A, 569, L4

Clayton, G. C. 1996, PASP, 108, 225

Clayton, G. C. 2012, JAAVSO, 40, 539

Clayton, G. C., \& Ayres, T. R. 2001, ApJ, 560, 986

Clayton, G. C., Geballe, T. R., \& Zhang, W. 2013, AJ, 146, 23

Ducati, J. R. 2002, VizieR Online Data Catalog: II/237

García-Hernández, D. A., Kameswara Rao, N., \& Lambert, D. L. 2011a, ApJ, 729,126

García-Hernández, D. A., Rao, N. K., \& Lambert, D. L. 2011b, ApJ, 739, 37

Iben, Jr., I., Tutukov, A. V., \& Yungelson, L. R. 1996, ApJ, 456, 750

Jeffery, C. S., \& Heber, U. 1993, A\&A, 270, 167

Jura, M., Balm, S. P., \& Kahane, C. 1995, ApJ, 453, 721

Men'shchikov, A. B., Schertl, D., Tuthill, P. G., Weigelt, G., \& Yungelson, L. R. 2002, A\&A, 393, 867

Modigliani, A., Goldoni, P., Royer, F., et al. 2010, in Observatory Operations: Strategies, Processes, and Systems III, Proc. SPIE, 7737, 773728

Rao, N. K., \& Lambert, D. L. 1993a, AJ, 105, 1915

Rao, N. K., \& Lambert, D. L. 1993b, MNRAS, 263, L27

Saio, H., \& Jeffery, C. S. 2002, MNRAS, 333, 121

Samus', N. N., Goranskii, V. P., Durlevich, O. V., et al. 2003, Astron. Lett., 29, 468

Sarre, P. J., Miles, J. R., \& Scarrott, S. M. 1995, Science, 269, 674

Scarrott, S. M., Watkin, S., Miles, J. R., \& Sarre, P. J. 1992, MNRAS, 255, 11P

Schmidt, G. D., \& Witt, A. N. 1991, ApJ, 383, 698

Sellgren, K., Werner, M. W., Ingalls, J. G., et al. 2010, ApJ, 722, L54

Tisserand, P., Wood, P. R., Marquette, J. B., et al. 2009, A\&A, 501, 985

Tisserand, P., Clayton, G. C., Welch, D. L., et al. 2013, A\&A, 551, A77

Vacca, W. D., Cushing, M. C., \& Rayner, J. T. 2003, PASP, 115, 389

Van Winckel, H., Cohen, M., \& Gull, T. R. 2002, A\&A, 390, 147

Vernet, J., Dekker, H., D’Odorico, S., et al. 2011, A\&A, 536, A105

Wehres, N., Linnartz, H., van Winckel, H., \& Tielens, A. G. G. M. 2011, A\&A, 533, A28

1 http://leonid.arc.nasa.gov/DIBcatalog.html 


\section{Appendix A: Position-velocity diagrams}
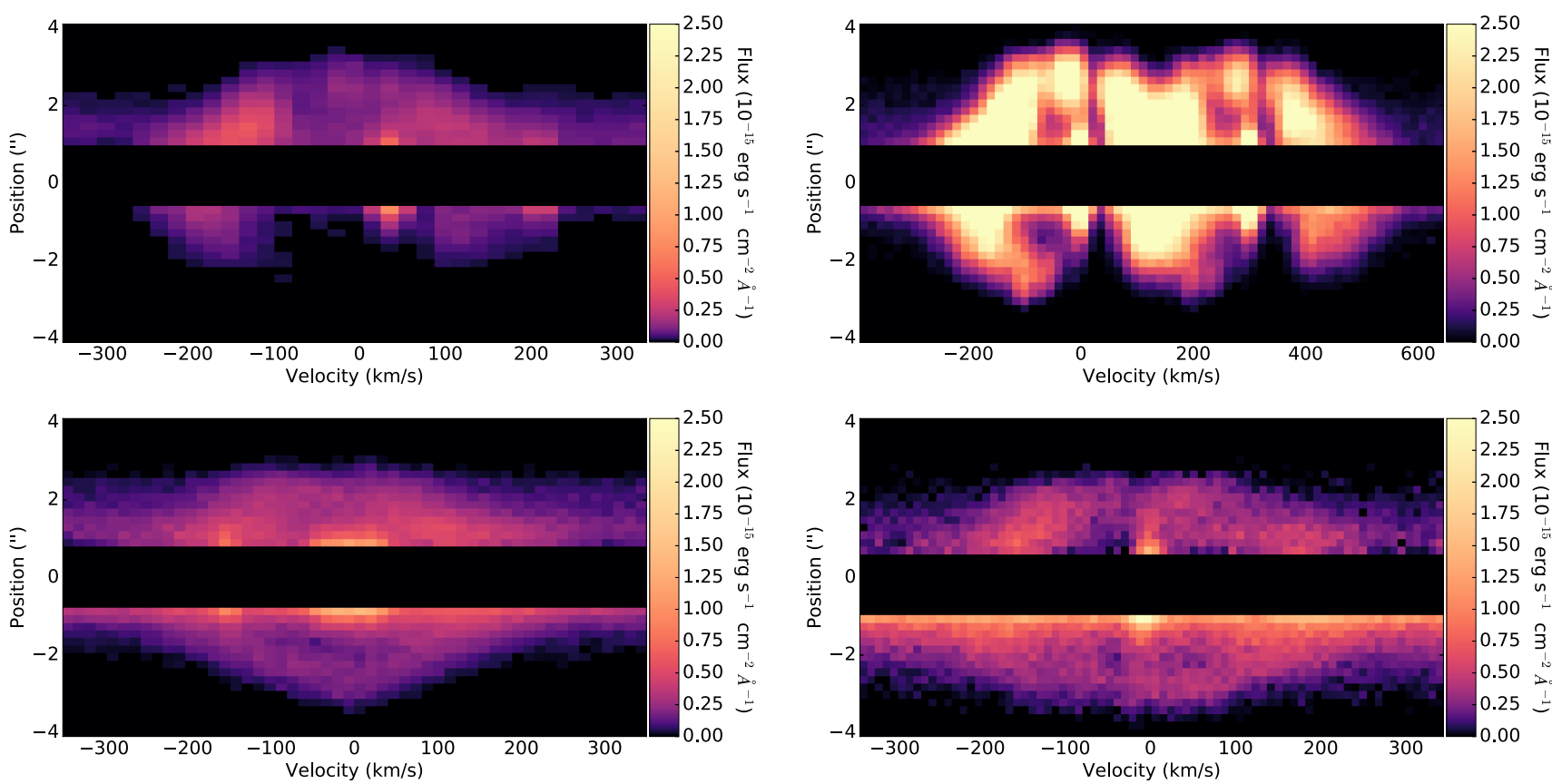

Fig. A.1. Position-velocity diagrams of $\mathrm{CH}^{+}$(top left), $\mathrm{Na} \mathrm{I} \mathrm{D} \mathrm{(top} \mathrm{right),} \mathrm{H} \alpha$ (bottom left) and the [C I] line at $9850 \AA$ A (bottom right) in V854 Cen. Both $\mathrm{CH}^{+}$and $\mathrm{Na}$ I D show a shape very similar to Ca II K (see main text); however, both Na I D components have a strong zero-velocity component. Note that the narrow emission line in the $\mathrm{CH}^{+}$diagram at $\sim 50 \mathrm{~km} \mathrm{~s}^{-1}$ is likely due to $\mathrm{Na}$ II. $\mathrm{H} \alpha$ and [C I] shows a similar, although less pronounced, behaviour.

\section{Appendix B: Unidentified emission feature spectra}
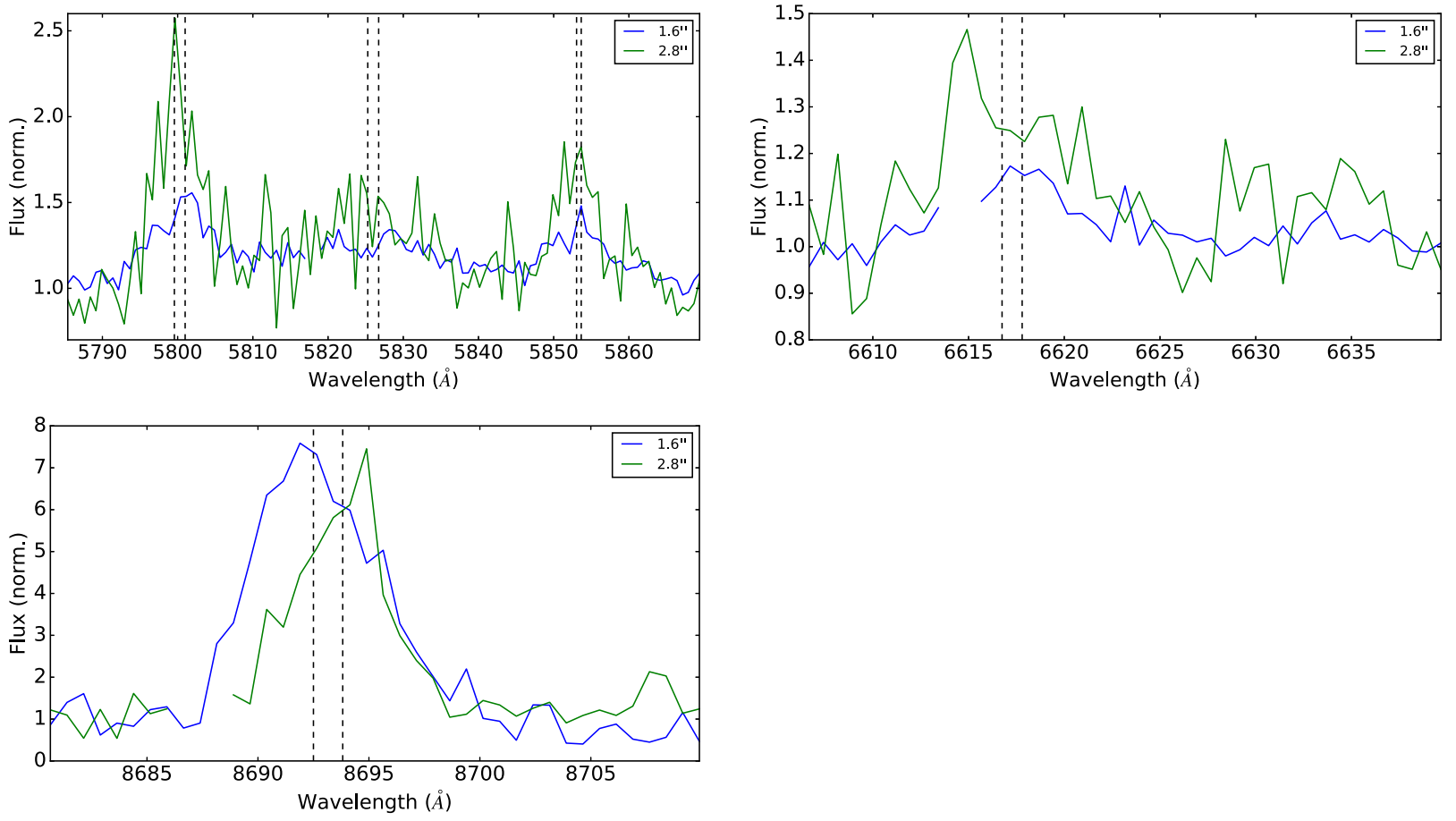

Fig. B.1. Spectra at 1.6" and 2.4" from the central object for all unidentified emission features. Dashed lines indicate the best-fit peak position for each feature. Top left: $\lambda \lambda 5800,5827$, and 5854. Top right: $\lambda 6617$. Bottom left: $\lambda 8692$. In all features except $\lambda 8692$, a blueshift with increasing distance from the central object is observed. The $\lambda 8692$ feature shifts towards longer wavelengths in the spectra shown here, but it shifts towards shorter wavelengths on the other side of the star. The $1.6^{\prime \prime}$ spectrum of $\lambda 8692$ has been stretched by a factor 8 for displaying purposes. 